\title{
VGCF detection of galaxy systems at intermediate redshifts ${ }^{\star}$
}

\author{
R. Barrena ${ }^{1}$, M. Ramella ${ }^{2}$, W. Boschin ${ }^{3,4}$, M. Nonino ${ }^{2}$, A. Biviano ${ }^{2}$, and E. Mediavilla ${ }^{1}$ \\ 1 Instituto de Astrofísica de Canarias, Vía Láctea s/n, 38200 La Laguna, Tenerife, Spain \\ e-mail: rbarrena@iac.es \\ 2 INAF, Osservatorio Astronomico di Trieste, via G.B. Tiepolo 11, 34131 Trieste, Italy \\ 3 INAF, Telescopio Nazionale Galileo, Roque de Los Muchachos, PO box 565, 38700 Santa Cruz de La Palma, Spain \\ 4 Dipartimento di Astronomia of the Università degli Studi di Trieste, via G.B. Tiepolo 11, 34131 Trieste, Italy
}

Received 17 May 2005 / Accepted 10 August 2005

\section{ABSTRACT}

We continue the development of our Voronoi Galaxy Cluster Finder (VGCF) technique by applying it to galaxy catalogs obtained with $B$ and $R$ band observations of four high galactic latitude fields of $0.5 \times 0.5$ square degrees each. These fields are deep $\left(R_{\lim } \sim 23, B_{\text {lim }} \sim 26\right)$ and partially overlap the Palomar Distant Cluster Survey (PDCS) fields at $0^{\mathrm{h}}$ and $2^{\mathrm{h}}$. We run the VGCF also on the original $V$ and $I$ band PDCS galaxy catalogs.

We identify a total of 48 clusters that are particularly reliable, being detected in at least two bands. The analysis of color-magnitude diagrams and, in a few cases, spectroscopic observations allow us to further increase the reliability of 25 of the 48 clusters. For these 26 clusters we also estimate redshifts that fall in the approximate range $0.2<z<0.6$.

We detect 41 VGCF clusters within the strict limits of the PDCS fields at $0^{\mathrm{h}}$ and $2^{\mathrm{h}}$. The PDCS catalog for the same regions consists of 28 clusters. The two catalogs have 20 clusters in common. These clusters together with the remaining PDCS and VGCF clusters lead to a total number of 46 "independent" clusters. The total number of clusters is therefore $20 \%$ larger than the number of VGCF clusters and more than $60 \%$ larger than the number of PDCS clusters. These results confirm a) that the VGCF is a competitive algorithm for the identification of optical clusters, and b) that a combined catalog of matched-filter and VGCF clusters constitutes a significant progress toward a more complete selection of clusters from bidimensional optical data.

Key words. galaxies: clusters: general - galaxies: distances and redshifts

\section{Introduction}

Clusters of galaxies play several roles in modern astrophysics. For example, they are laboratories to test theories of galaxy formation and evolution. They are probes of large scale structure. They are gravitational telescopes enabling us to see distant objects that would otherwise not be detectable. Clusters also provide powerful constraints on cosmological models. It is therefore not surprising that so many efforts have been, and are being spent to identify clusters of galaxies. Ideally one would like to identify clusters with a well-defined selection criterion over the widest redshift range including clusters with the widest range of properties.

In practice, there are two main methods to detect clusters. The first, classical technique, based on optical/NIR observations, consists of detecting clusters by discovering galaxy concentrations with respect to the mean galaxy background/foreground density. The second one, based on observations in the soft X-ray band (0.1-10 keV), consists of detecting

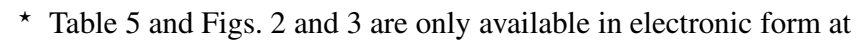
http://www . edpsciences.org clusters by observing the X-ray emission from the hot intracluster medium.

Both optical and X-ray selected samples have advantages and difficulties. In the last 10-15 years X-ray cluster surveys had a strong development (see, e.g., Rosati et al. 1998; Boschin 2002; Moretti et al. 2004 for recent surveys) mainly thanks to the ROSAT, XMM and Chandra satellites. The main advantage of X-ray surveys is that clusters look sharper in the X-ray sky than in the optical sky. The background is low in X-ray observations and the $\mathrm{X}$-ray cluster emission is proportional to the square of the local gas density.

Unfortunately low mass systems, whose X-ray emission is generally weak, are very difficult to detect, even at low redshifts. Furthermore, there may be clusters of similar optical richness but different ICM histories leading to different X-ray luminosities (Gilbank et al. 2004; Donahue et al. 2001). In view of these problems it is interesting to identify clusters from the galaxy distribution in optical images.

In this paper we are concerned with optical identifications. They have a strong advantage with respect to X-ray identifications: the availability of a large number of large aperture ground telescopes with high quantum efficiency detectors. 
In the last decade several deep imaging cluster surveys have been performed. Notable examples include the Palomar Distant Cluster Survey (PDCS, Postman et al. 1996), the ESO Imaging Survey (EIS, Nonino et al. 1999; Olsen et al. 1999), the driftscan survey of Zaritsky et al. 1997, the KPNO Deeprange (Postman et al. 2002). These surveys are typically designed to find rich clusters at high redshift within deep galaxy surveys of relatively small sky coverage.

Wide field galaxy surveys based on the digitization of photographic plates have produced (e.g. Dalton et al. 1997; Lumsden et al. 1992), and are still producing (e.g. Lopes et al. 2004), impressive results. However, modern wide field imaging is becoming increasingly common since large format CCD cameras are now available at several telescopes. These new instruments allow a systematic search for medium-high redshift galaxy clusters within photometric catalogs covering wide areas of the sky.

The Sloan Digital Sky Survey, SDSS (http://www. sdss. org/+), has been used so far to produce two cluster catalogs (Goto et al. 2002; Bahcall et al. 2003). Other notable cluster catalogs based on wide-field surveys are the Stanford Cluster Search (Willick et al. 2001, StaCS), the Toronto RedSequence Cluster Survey (Gladders \& Yee 2000, 2005), and the Las Campanas Distant Cluster Survey (Gonzalez et al. 2001, LCDCS).

Since there is a significant background in optical when searching for clusters (especially those at intermediate and high redshifts), galaxy systems are identified within galaxy surveys using several techniques which selectively suppress the background. They are mainly based on two different algorithms (see Kim et al. 2002): the matched-filter algorithm (Postman et al. 1996), hereafter PLG96) and the Voronoi tessellation algorithm (Ramella et al. 2001).

Among other techniques there are the cut-and-enhance method (Goto et al. 2002) and the cluster identification based on the detection in color space of the red sequence of earlytype galaxies at the core of (rich) clusters (Gladders \& Yee 2000).

In a previous paper (Ramella et al. 2001, hereafter R01) we propose the VGCF, a procedure based on the Voronoi tessellation to identify clusters within photometric galaxy catalogs. As we specify in R01, the procedure is meant to complement other techniques, in particular the matched filter of PLG96. The matched filter algorithm is powerful because it is an optimal filtering technique that can detect clusters that are weak overdensities of the angular galaxy density distribution. No technique is perfect: the matched filter algorithm can miss clusters because of its model-dependency and because of the smoothing of the data that "washes out" small structures in the periphery of large overdensities. The VGCF has among its strengths the fact of being model independent and the lack of smoothing. On the other hand, some of the matched filter clusters would never be detectable by the VGCF because of their low signalto-noise ratio (SNR) and/or because of their lack of a "cusp" in their density profile. The combined use of both techniques should lead to a significant improvement of the completeness of optically selected cluster catalogs.
Table 1. Log of the observations with WFC@INT.

\begin{tabular}{llccc}
\hline \hline Date & Field & $t_{\exp }(\mathrm{s})$ & Seeing & Airmass \\
\hline \multicolumn{5}{c}{$B_{\mathrm{H}}$ band } \\
$99-10-13$ & F0028+0515 & 10000 & $1.6^{\prime \prime}$ & $1.8-1.1$ \\
$99-10-14$ & F0027+0555 & 10000 & $1.6^{\prime \prime}$ & $1.2-1.1$ \\
$99-10-13$ & F0228+0115 & 10000 & $1.7^{\prime \prime}$ & $1.4-1.2$ \\
$99-10-14$ & F0226+0106 & 10000 & $1.1^{\prime \prime}$ & $1.6-1.1$ \\
\hline \multicolumn{5}{c}{$R_{\mathrm{H}}$ band } \\
$99-10-12$ & F0028+0515 & 3600 & $1.4^{\prime \prime}$ & $1.8-1.2$ \\
$99-10-14$ & F0027+0555 & 4200 & $1.6^{\prime \prime}$ & $1.9-1.2$ \\
$99-10-12$ & F0228+0115 & 4800 & $1.2^{\prime \prime}$ & $1.7-1.3$ \\
$99-10-13$ & F0226+0106 & 6000 & $1.4^{\prime \prime}$ & $1.5-1.1$ \\
\hline
\end{tabular}

Here we follow up on our previous work on the VGCF (R01) and discuss its performance on our own multi-band photometric data, as well as on PDCS data.

In Sect. 2 we describe observations and image reduction techniques. In Sect. 3 we introduce the cluster detection technique and present our catalog of clusters in Sect. 4. In Sect. 5 we study color properties of clusters and, for a subsample, we also estimate photometric redshifts. In Sect. 6 we compare our VGCF catalog to the PDCS catalog. We present the summary of our work in Sect. 7.

Throughout this paper $\Omega_{\mathrm{m}}=0.3, \Omega_{\Lambda}=0.7$ and $H_{0}=$ $70 \mathrm{~km} \mathrm{~s}^{-1} \mathrm{Mpc}$ are used.

\section{Observations and galaxy catalogs}

Our aim is to test the performances of the VGCF (R01) on new multi-band observations where images could help our understanding of the nature of the VGCF detections. At the same time we want to improve the comparison with the PDCS cata$\log$, generated with a matched-filter algorithm, in order to verify that we can significantly increase the completeness of cluster catalogs by using both search methods.

We select 4 pointings for the Wide Field Camera (WFC) mounted at the prime focus of the $2.5 \mathrm{~m}$ Isaac Newton Telescope (INT), located at the Roque de Los Muchachos Observatory, La Palma. We carried out observations from October 12 to October 14, 1999 using the $B_{\mathrm{H}}$ and $R_{\mathrm{H}}$ Harris filters. The $\log$ of our observations is given in Table 1 . We list the coordinates of our pointings in Table 2 . The field of view of the WFC is $34 \times 34$ square minutes and the scale is $0.333^{\prime \prime} /$ pixel. With this field of view, our pointings allow us to observe 12 PDCS clusters (PLG96) located in the PDCS fields at $0^{\mathrm{h}}$ and $2^{\mathrm{h}}$ (hereafter PDCSO and PDCS2, respectively). The properties of these clusters are representative of those of the whole PDCS catalog. The partial overlap of two of our pointings allows us to check the consistency of our photometric and astrometric reductions. It also allows us to check of the stability of our clustering analysis.

We perform multiple integrations in the direction of each pointing. We offset each integration by about $20^{\prime \prime}$. The dithering pattern of the integrations allows us to create a "supersky" that we use to correct our images for fringing patterns (e.g. Gullixson 1992). The dithering also allows us to clean cosmic 
Table 2. Coordinates and areas of INT pointings and PDCS fields.

\begin{tabular}{lcccc}
\hline \hline Field & $\alpha_{2000}$ & $\delta_{2000}$ & \multicolumn{2}{c}{ Area (sq. deg.) } \\
\hline & & & $B_{\mathrm{H}}$ & $R_{\mathrm{H}}$ \\
F0028+0515 & $00: 28: 50$ & $05: 15: 20$ & 0.277 & 0.284 \\
F0027+0555 & $00: 27: 25$ & $05: 55: 35$ & 0.296 & 0.321 \\
F0228+0115 & $02: 28: 05$ & $01: 15: 30$ & 0.285 & 0.303 \\
F0226+0106 & $02: 26: 25$ & $01: 06: 20$ & 0.325 & 0.294 \\
\hline & & & $V_{4}$ & $I_{4}$ \\
PDCS 0 & $00: 29: 11$ & $05: 31: 55$ & 1.074 & 0.970 \\
PDCS 2 & $02: 28: 33$ & $00: 55: 45$ & 1.120 & 0.927 \\
\hline
\end{tabular}

rays and to avoid gaps between the CCDs of the WFC in the final images.

We perform a standard reduction of our WFC observations with the $\operatorname{IRAF}^{1}$ package. We reduce each CCD frame separately. We also find astrometric solutions for each frame separately using the $\mathrm{USNO}^{2}$ catalogs.

We coadd the different dithering frames into a single image using our own routines. Based on the USNO catalogs, the accuracy of our astrometry is $\sim 0.5^{\prime \prime}$ over the full field of the WFC without significant aberrations in the PSF. The mean ellipticity of the PSF is 0.11 over the whole WFC field. Residual PSF elongations have no preferred direction.

Because our three nights at the INT were not photometric, we calibrate our WFC images with observations obtained with the $1 \mathrm{~m}$ Jacobus Kaptein Telescope (JKT; Roque de los Muchachos Observatory) during the night of September 22, 2000. We select one pointing for each of our INT frames. The criterion for the selection of the pointings is to have enough non-saturated stars and galaxies in the WFC images in the magnitude range $R=17-19$. Exposure times of $30 \mathrm{~min}$ at the JKT give good SNR images in this magnitude range. We typically observe 20-30 objects (both stars and galaxies) per field.

We perform AUTOMAG aperture photometry on standard stars using the SExtractor photometry package (Bertin \& Arnouts 1996). With this process we obtain the instrumental magnitudes $\left(m_{\lambda, \text { ins }}\right)$. The long exposure time of the scientific images (of the order of $10000 \mathrm{~s}$ ) does not allow an estimate of an airmass for the coadded images. For this reason, we can only determine a reduced transformation for photometric calibrations by neglecting the color term. The reduced transformations have the form $m_{\lambda}=m_{\lambda \text {,ins }}+b_{\lambda}$, where $m_{\lambda}$ is the tabulated magnitude of the star in each band $\lambda$ and $b_{\lambda}$ is the offset magnitude.

We finally identify galaxies in our $B_{\mathrm{H}}$ and $R_{\mathrm{H}}$ images and measure their magnitudes with the SExtractor package. To these catalogs of galaxies, we add those in the $V_{4}$ and $I_{4}$ bands kindly provided to us by M. Postman.

As a final step, we transform all magnitudes into the Johnson-Cousins system (Johnson \& Morgan 1953; Cousins 1976).

\footnotetext{
${ }^{1}$ IRAF: Image Reduction and Analysis Facility distributed by National Optical Astronomy Observatories.

${ }^{2}$ United States Naval Observatory, version 1.0.
}

Table 3. Completeness and limiting magnitudes.

\begin{tabular}{lcccc}
\hline \hline Field & \multicolumn{2}{c}{$m_{\mathrm{c}}$} & \multicolumn{2}{c}{$m_{\lim }$} \\
\hline & $B$ & $R$ & $B$ & $R$ \\
F0028+0515 & 24.4 & 21.8 & 26.0 & 23.1 \\
F0027+0555 & 24.5 & 22.9 & 26.2 & 24.2 \\
F0228+0115 & 24.4 & 22.3 & 25.8 & 23.8 \\
F0226+0106 & 23.4 & 21.3 & 24.7 & 23.3 \\
\hline \multirow{2}{*}{$\begin{array}{c}\text { PDCS 0 } \\
\text { h }\end{array}$} & 23.5 & 21.0 & $V$ & $I$ \\
PDCS 2 $^{\mathrm{h}}$ & 23.5 & 21.2 & 25.1 & 22.8 \\
\hline
\end{tabular}

For $B$ and $R$ we use $B=B_{\mathrm{H}}+0.13$ and $R=R_{\mathrm{H}}$, derived from the Harris filter characterization (http://www. ast.cam.ac.uk/ wfcsur/technical/photom/colors/) and assuming a $B-V \sim 1.0$ for E-type galaxies (Poggianti 1997).

For $V$ and $I$ we use the transformations given in PLG96.

Because the PDCS fields are at high galactic latitude, galactic extinction is low. We estimate $A_{B} \sim 0.09, A_{V} \sim 0.06$, $A_{R} \sim 0.04$ and $A_{I} \sim 0.03$ from Burstein \& Heiles (1982) reddening maps.

Thus, we have four galaxy catalogs, one for each (JohnsonCousins) photometric band $B, V, R$ and $I$. We list in Table 3 the completeness magnitude, $m_{\mathrm{c}}$, and limiting magnitude $m_{\mathrm{lim}}$, of these four catalogs. Completeness and limiting magnitudes correspond to 5- $\sigma$ and 3- $\sigma$ source detections respectively.

As a part of this project we also obtained spectroscopic data using the Device Optimized for the Low Resolution (DOLoRes) at the Telescopio Nazionale Galileo (TNG). We used DOLoRes both in Multi-Object Spectroscopy (MOS) mode and in Long Slit mode. The size of the MOS fields is $6 \times 9 \operatorname{arcmin}^{2}$ on a Loral $2048 \times 2048 \mathrm{CCD}$ with $15 \mu$ pixels. We used the LR-B grism providing a resolution of $2.8 \AA /$ pix and a wavelength coverage from $3000 \AA$ to $8800 \AA$. With integration times of $90 \mathrm{~min}$ and with an average seeing of $1.5^{\prime \prime}$, we obtained spectra for 142 targets with 4 masks and slits 1.1" wide. The SNR are in the range 5 to 10 for targets with magnitudes as deep as $R \simeq 21$. We use the same set-up also in Long Slit mode, although in this case we widen the slit up to $1.5^{\prime \prime}$ because of the poorer seeing $\left(\sim 1.5^{\prime \prime}\right)$. Total exposure times in Long Slit mode are of $45 \mathrm{~min}$.

We reduce the TNG and CFHT data using the standard procedures of the IRAF package. We perform cosmic ray rejection, sky subtraction, aperture extraction and wavelength calibration. In most cases the SNR ranges from 5 to 10 , or even exceeds these values. We measure the redshift using the crosscorrelation technique xcsao (Tonry \& Davis 1979) implemented in the RVSAO package (developed at the Smithsonian Astrophysical Observatory Telescope Data Center). We use as templates the spectra of Elliptical, S0, Sa, Sb, Sc and Irregular type galaxies taken from Kinney et al. (1996).

\section{Detecting clusters with the VGCF}

In order to identify clusters of galaxies in our galaxy catalogs we use the VGCF. This technique is described in detail 
in R01. Here we briefly summarize the main characteristics of the VGCF.

The VGCF uses the Voronoi tessellation in order to assign to each object a local density given by the inverse of the area of the Voronoi tessel of that point. We remind that a Voronoi tessellation of a two-dimensional distribution of galaxies is a unique plane partition into convex cells, each of them containing one, and only one object.

Then the VGCF determines the background density of objects by fitting the low-density end of the observed integral density distribution.

The VGCF compares the observed density distribution with the empirical density distribution expected for a Poissonian distribution of points having the same density as derived from the fit to the low-density end of the observed integral density distribution.

According to Kiang (1966), the expected background distribution has the form:

$\mathrm{d} p(\tilde{a})=\frac{4^{4}}{\Gamma(4)} \tilde{a}^{3} \mathrm{e}^{-4 \tilde{a}} \mathrm{~d} \tilde{a}$

where $\tilde{a} \equiv a /\langle a\rangle$ is the cell area in units of the average cell area $\langle a\rangle$. As each cell contains exactly one galaxy, the corresponding density is the inverse of the cell area $f \equiv 1 / a$.

The user establishes a density threshold above the Poissonian distribution, i.e. a minimum confidence level for significant overdensities. Here, as in R01, we set this threshold at the $80 \%$ level.

The algorithm then defines overdense regions as those composed by adjacent Voronoi cells with a density higher than the chosen threshold. By computing the probability that an overdensity corresponds to a background fluctuation (see R01), the VGCF discards overdensities with probabilities greater than a given threshold. We set this threshold at the $95 \%$ level.

The VGCF regularizes the shape of the overdense regions. First, it assumes that all the points inside the convex hull defined by the set of points belong to the overdensity itself. Next, it fits a circle to the overdense region and expands it until the mean density inside the circle is lower than the density of the original region.

The output of the VGCF is a catalog of overdensities, or clusters, listed with their main characteristics.

The VGCF does not assume density or luminosity profiles for clusters and does not smooth the data. The VGCF can identify clusters irrespective of their shape and is only weakly affected by edge effects and by "holes" in the galaxy distribution (e.g. those caused by extremely bright stars). The algorithm is fast, and automatically assigns members to the structures.

Because of the depth of our galaxy catalogs, and the corresponding high galaxy projected number densities, it is necessary to run the VGCF in magnitude bins, as in R01.

The choice of the bin width cannot be equally optimal for the detection of all clusters. Following R01, we use bins that are 2 mag wide. R01 gage their bins for cluster catalogs covering a redshift range very close to that of our present sample.

We select galaxies within a magnitude bin and run the VGCF over this sub-sample of galaxies. We then shift the
Table 4. Details of the magnitude bins of the VGCF runs.

\begin{tabular}{ccccc}
\hline \hline Field $^{a}$ & First bin & Last bin & First bin & Last bin \\
\hline \multicolumn{4}{c}{ Catalog in $B$ band } & \multicolumn{2}{c}{ Catalogs in $R$ band } \\
F0028 & $20.0-22.0$ & $22.5-24.5$ & $18.0-20.0$ & $20.5-22.5$ \\
F0027 & $20.0-22.0$ & $22.5-24.5$ & $19.0-21.0$ & $21.5-23.5$ \\
F0228 & $20.0-22.0$ & $22.5-24.5$ & $18.0-20.0$ & $20.5-22.5$ \\
F0226 & $20.0-22.0$ & $22.5-24.5$ & $17.5-19.5$ & $20.0-22.0$ \\
\hline \multicolumn{4}{c}{ Cataloger in $V$ band } & Cataloger in $I$ band \\
PDCS0 & $18.0-20.0$ & $21.5-23.5$ & $17.0-19.0$ & $19.5-21.5$ \\
PDCS2 & $18.5-20.5$ & $21.5-23.5$ & $17.0-19.0$ & $19.5-21.5$ \\
\hline
\end{tabular}

${ }^{a}$ F0028, F0027, F0228 and F0226 correspond to F0028+0515, F0027+0555, F0228+0115 and F0226+0106 fields, respectively.

magnitude bin faintward by 0.1 mag and run the VGCF again. The width of the step is the same as in R01.

We select the first bin starting from fainter magnitudes than those of the brightest galaxies. This is necessary in order to have enough counts in the first bin for a correct estimate of the background galaxy density. The omission of the (few) brightest galaxies has no impact on our analysis. The brightest and faintest magnitudes of our binning procedure are summarized in Table 4. We obtain at least 25 catalogs in each band.

In order to "merge" into a single cluster sequences of overlapping overdensities identified in different magnitude bins (in the same band), we use similar criteria to those indicated by $\mathrm{R} 01$. The slight variations are dictated by the different data sets at our disposal. In practice, we proceed as follows.

We consider a set of overdensities a cluster if there are at least 4 overdensities that: a) have centers that are closer than $15^{\prime \prime}$ from the corresponding overdensity in the next magnitude bin, b) are replicated within at least 4 mag bins that are adjacent or at most with a total of one bin missing. We then require the cluster to be identified in at least two photometric bands with centers separated by less than $15^{\prime \prime}$.

By applying these criteria to the VGCF runs in magnitude bins on our BVRI catalogs we obtain a final list of 48 clusters.

\section{The cluster catalog}

We present the catalog of the 48 VGCF clusters in Table 5. We give the cluster ID in Col. (1). In Cols. (2), (3), (4) and (5) we list the SNRs in the $B, V, R$ and $I$ bands respectively. We estimate the SNR as the number of members within the area of the overdensity divided by the square root of background counts expected within the same area according to the VGCF background fit. The SNR values in Cols. (2)-(5) are averages over all overdensities associated with the cluster (in each band). A " 0 " indicates that the cluster has not been identified in the corresponding band and a blank space indicates that we have no data at that position.

In Cols. (6) and (7) we list J2000 right-ascension and declination, respectively. In Col. (8) we give the angular radius of the cluster (in arcsec). The value we list is the average of the radii of the detections in all bands. We list our photometric redshift estimate in Col. (9). For the clusters with both $B R$ and $V I$ redshift estimates, the value we give in the table is the average of the two estimates. For clusters that have a PDCS 

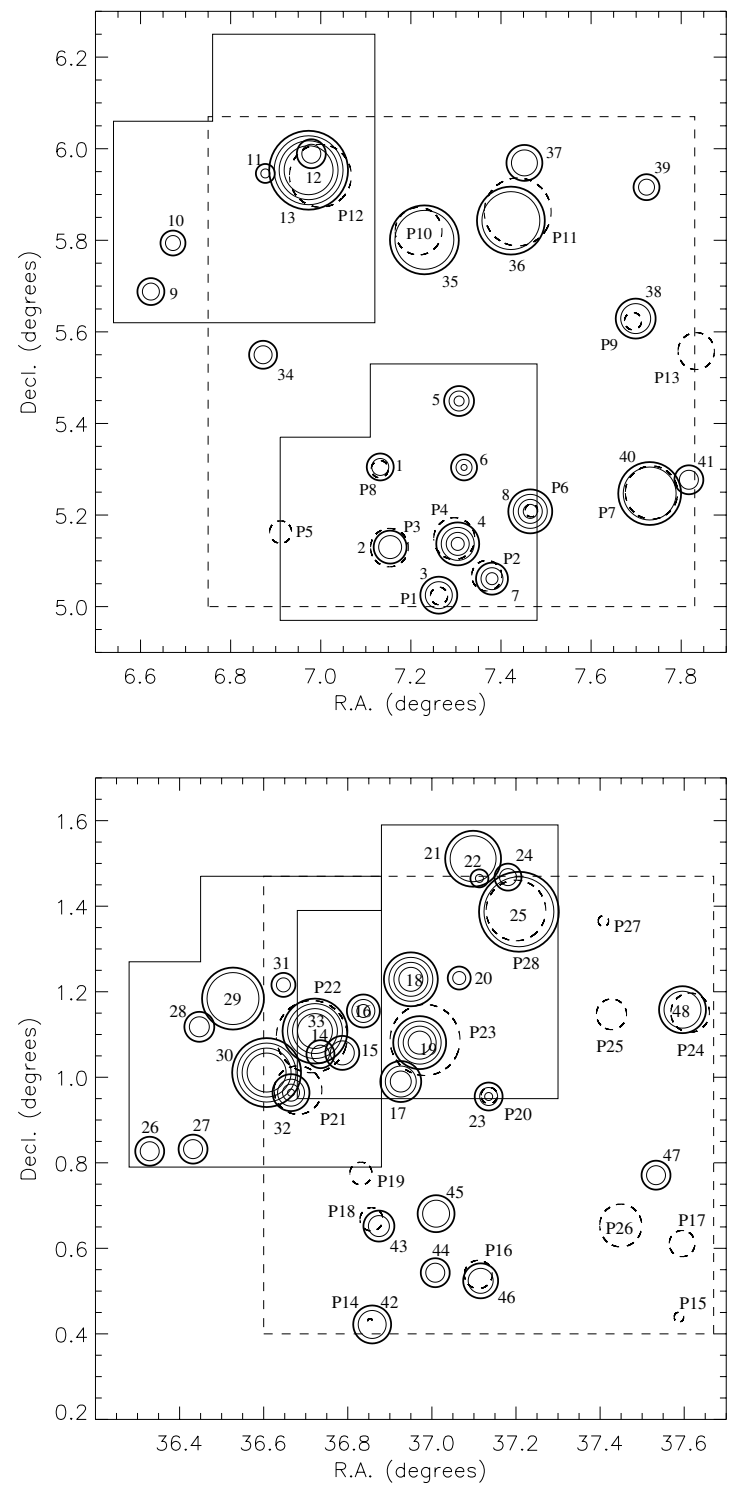

Fig. 1. VGCF and PDCS clusters. We draw with a thick line our clusters, with a dashed line PDCS clusters, with a thin line the VGCF detections in individual bands (the radii of these last detections are arbitrary. VGCF clusters are labeled with their ID number, PDCS clusters are labeled with A "P" followed by the PDCS identification number.

counterpart we give the PDCS redshift estimate in Col. (10). We add comments in Col. (11).

Figure 1 is the graphical representation of Table 5. The dashed square regions represent the PDCS fields. The thin solid lines delimit our $B$ and $R$ survey, i.e. fields F0028+0515, $\mathrm{F} 0027+0555$, F0228+0115, and F0226+0026. Circles drawn with thick lines are our clusters. Radii correspond to those listed in Table 5. Circles drawn with thin lines represent detections in individual bands. The radius of these circles is arbitrary. The label next to each circle is the cluster ID number in Table 5. Dashed line circles are the original PDCS clusters. The radii of these circles are those given by PLG96. We label these circles with a "P" followed by the PDCS identification number.

As far as the overlap region between the two fields F0228+0115 and F0226+0106 is concerned, it provides a check of the reliability of the detections. This check is important because of the different structure of the galaxy distribution in the two fields. In principle, this difference may cause the VGCF to estimate slightly different background levels leading to inconsistencies in the overlap region. Reassuringly, we identify the same set of clusters in both frames within the overlap region. In Table 5 the properties of the clusters in the overlap region are averages of the properties of the clusters identified within each frame.

\section{Color analysis of cluster candidates}

In this section we use our $B V R I$ photometry to find evidence that our clusters are real physical systems. We analyze the Color-Magnitude Diagrams (CMD) of each cluster, and try to identify a Sequence of Early Type galaxies (ETS).

Almost all rich clusters have a sequence of early-type galaxies easily distinguishable in a CMD (Gladders \& Yee 2000, and references therein). The ETS also gives the possibility of estimating the redshift of clusters (Gladders \& Yee 2005)

In order to identify the ETS in our CMD, we start from the ETS of Coma $(z=0.023)$ for the $(B-R, R)$ CMD and from the ETS of A118 $(z=0.31)$ for the $(V-I, I)$ CMD. Secker et al. 1997 show that the ETS of Coma follows the relation $(B-R)=(-0.056 \pm 0.002) R+(2.41 \pm 0.04)$. We fit the ETS of A118 from galaxy members of this cluster (Busarello et al. 2002). In this case, the red sequence follows the relation $(V-I)=(-0.060 \pm 0.007) I+(2.77 \pm 0.09)$.

We then compute the set of ETS that Coma and A118 would have if they were were moved at the redshifts $z=$ $0.1,0.2,0.3 \ldots 0.7$. We compute the expected ETS by applying to the observed ETS theoretical evolutionary- and K-corrections for a passively evolving population of early-type galaxies (Poggianti 1997). We use these grids of semi-empirical ETS to guide our eye in the detection of possible sequences in the $(B-R, R)$ and $(V-I, I) \mathrm{CMD}$.

Because several of our clusters are rather poor, the impact of foreground/background interlopers on the identification of ETS may be significant. For this reason we apply a statistical technique to clean the CMD. In particular, we perform a statistical subtraction of the galaxy background using the method proposed by Phelps (1997). He uses his method to clean CMD of star clusters. We find it to also clean quite effectively the CMD of our galaxy clusters.

In practice, we proceed as follows. Next to the cluster under study we select a region (field) of the sky without any cluster identification. In order to have sufficient statistics, the area of the field is five times larger than the area containing the cluster under study.

We divide both the CMD of the cluster and of the field into a grid of $20 \times 20$ square cells with a side of 0.2 mag. We then eliminate one randomly selected object from each cell of the cluster CMD every five objects present in the same cell of the CMD of the field.

When we find an ETS, we use it to derive an approximate indication of the photometric redshift of the cluster. The $(B-R$, $R$ ) ETS significantly shifts toward redder colors in the redshift 
range $0.0<z<0.3$. The $(V-I, I)$ ETS shifts at the highest rate in the redshift range $0.3<z<0.5$. Our $B, V, R$ and $I$ bands are appropriate for estimating photometric redshifts $z<0.5$. Redshift estimates of clusters with $0.5<z<0.7$ will be quite crude.

In order to estimate the photometric redshift we select galaxies within a color range $\pm 0.3 \mathrm{mag}$ from the ETS that we identify with the aid of the semi-empirical grid of ETS. We then fit a new ETS to these galaxies. We constrain the slope of the ETS to be the same as that of the Coma cluster in the $(B-R$, $R) \mathrm{CMD}$ and of $\mathrm{A} 118$ in the $(V-I, I) \mathrm{CMD}$.

In Fig. 2 we plot the CMD of the 7 clusters that present an ETS both in the $(B-R, R)$ and in the $(V-I, I)$ CMD. In Fig. 3 we plot the CMD of the 18 galaxy clusters with evidence of only one ETS in either their $(B-R, R)$ or $(V-I, I)$ diagram.

The redshift estimates range from $z_{\mathrm{ETS}}=0.12(\mathrm{~V} 31)$ to $z_{\text {ETS }}=0.75$ (V05). For each cluster in Figs. 2 and 3 we write the estimated redshift next to the fitted ETS. We also list all redshifts in Table 5 . In this table $z_{\text {phot }}$ is the average between two redshift estimates if a cluster has both $(B-R, R)$ ETS and $(V-I, I)$ ETS.

In Figs. 4 we show $R$ band images of cluster V05 (left panel) and cluster V10 (right panel). These two clusters are our most distant confirmed detections, $z=0.75$ and $z=0.6$, respectively. The central galaxies in these images correspond to brightest galaxies in the ETS of these systems.

External and systematic errors are likely to dominate the real uncertainty of our redshift estimates. Given the accuracy of the photometry, we can estimate $\delta(B-R)$ and $\delta(V-I)$ to be about 0.15 mag for objects with $R=20$. These errors produce a redshift uncertainty of about $\delta z=0.1$.

An error of $\delta z=0.1$ in the photometric redshifts allows us to distinguish between "nearby" and "distant" clusters and it is comparable to the redshift estimate output by the matched-filter algorithm for PDCS clusters.

\subsection{Spectroscopic confirmation of photometric redshifts}

We present here follow up observations and archival data to improve/verify our ability to identify real physical clusters, their possible ETS and the reliability of the photometric redshift we derive from the ETS in the $B R$ and/or VI data.

We observed clusters V06 and V18 with the Device Optimized for the Low Resolution (DOLoRes) at the Telescopio Nazionale Galileo (TNG), in Multi-Object Spectroscopy (MOS) mode.

We retrieved spectra for V04 (P04) from the CFHT archive (see Adami et al. 2000 for details). We reduced the spectra and obtain new redshift measurements.

Finally, we found within SDSS redshifts of four galaxies, one in each of the following clusters: V17, V23, V30, and V48.

We list the redshifts we measure in confirmed clusters in Table 6 (cluster V18 is not confirmed spectroscopically).

We analyze in this section three further PDCS clusters P33 and P36 in the PDCS field at $9^{\mathrm{h}}$ and P63 in the PDCS field at $13^{\mathrm{h}}$. For these clusters we have both photometric and spectroscopic data. We include here the analysis of these clusters since their data at our disposal are comparable to those of the other clusters and therefore provide a further check of our procedures of confirmation and redshift estimation.

$B$ and $R$ photometry of clusters $\mathrm{P} 33, \mathrm{P} 36$, and $\mathrm{P} 63$ is from the HiRAC camera mounted at the $2.5 \mathrm{~m}$ Nordic Optical Telescope (NOT), located at Roque de los Muchachos Observatory, La Palma. We carried out these observations during three nights of February 2000 under very good photometric and seeing conditions using $B$ and $R$ Johnson filters (exposure times are $10000 \mathrm{~s}$ and $6000 \mathrm{~s}$ in the $B$ and $R$ band, respectively). Spectroscopy is from DOLoRes (used in Long Slit mode) at the TNG. We measured additional 18 redshifts for P33 from spectra retrieved from the CFHT archives.

We treat P33, P36 and P63 clusters in the same way as our VGCF clusters and identify an ETS in each of them. We plot the CDM of these three clusters in Fig. 5 and list the redshifts of their members in Table 6.

Finally, in order to test our photometric redshift estimates, we also use the spectroscopic redshifts of P11, P12 and P23 published in Holden et al. (1999).

With these last three redshifts we can compare photometric to spectroscopic redshifts for 13 clusters.

The spectroscopic survey of cluster V04 (P04) reveals a peak of 9 galaxies in the range $163000<c z<167000 \mathrm{~km} \mathrm{~s}^{-1}$. Applying the biweight estimator (Beers et al. 1990), we find that the cluster mean redshift is $z=0.5505 \pm 0.0004$. The 9 member galaxies are within $3^{\prime}$, that is, within $1.9 h^{-1} \mathrm{Mpc}$ from the center of the cluster. Based on these 9 galaxies, we compute a velocity dispersion $\sigma_{\mathrm{v}}=311_{-97}^{+171}$ in the cluster rest frame (Harrison \& Noonan 1979).

This cluster has been identified by both us and PLG96: its spectroscopic confirmation is reassuring. In particular it shows that even relatively poor clusters can be detected with either the VGCF or the matched-filter algorithm. Because we find no ETS in the CMD of this cluster, this cluster also clarifies a limit of our color confirmation technique: an ETS is clearly identifiable only in relatively rich clusters.

In the case of cluster V06 we find a peak along the lineof-sight and within $3^{\prime}\left(1.3 h^{-1} \mathrm{Mpc}\right)$ of the center. There are 9 galaxies in the range $107000<c z<112000 \mathrm{~km} \mathrm{~s}^{-1}$. Using the biweight estimator, we find that the cluster mean redshift is $z=0.366 \pm 0.001$. Its velocity dispersion is $\sigma_{\mathrm{v}}=$ $797_{-238}^{+408} \mathrm{~km} \mathrm{~s}^{-1}$ in the cluster rest frame.

The fact that we identify an ETS for V06 is consistent with the cluster velocity dispersion, typical of a richer system than V04. The (average) photometric redshift we obtain from the ETS is $z_{\text {phot }}=0.43$. There is a $\delta z \simeq 0.06$ difference between the photometric and spectroscopic redshifts, well within our estimated uncertainty of $\delta z \simeq 0.1$. The spectroscopic confirmation that V06 is a real physical system is important for the VGCF since V06 has not been identified by the matched-filter algorithm.

We are not able to identify a system along the line of sight of V18. Redshifts are spread over a wide redshift range and show no significant peak. The result of the spectroscopic observations is consistent with the fact that we do not find an ETS for V18. 

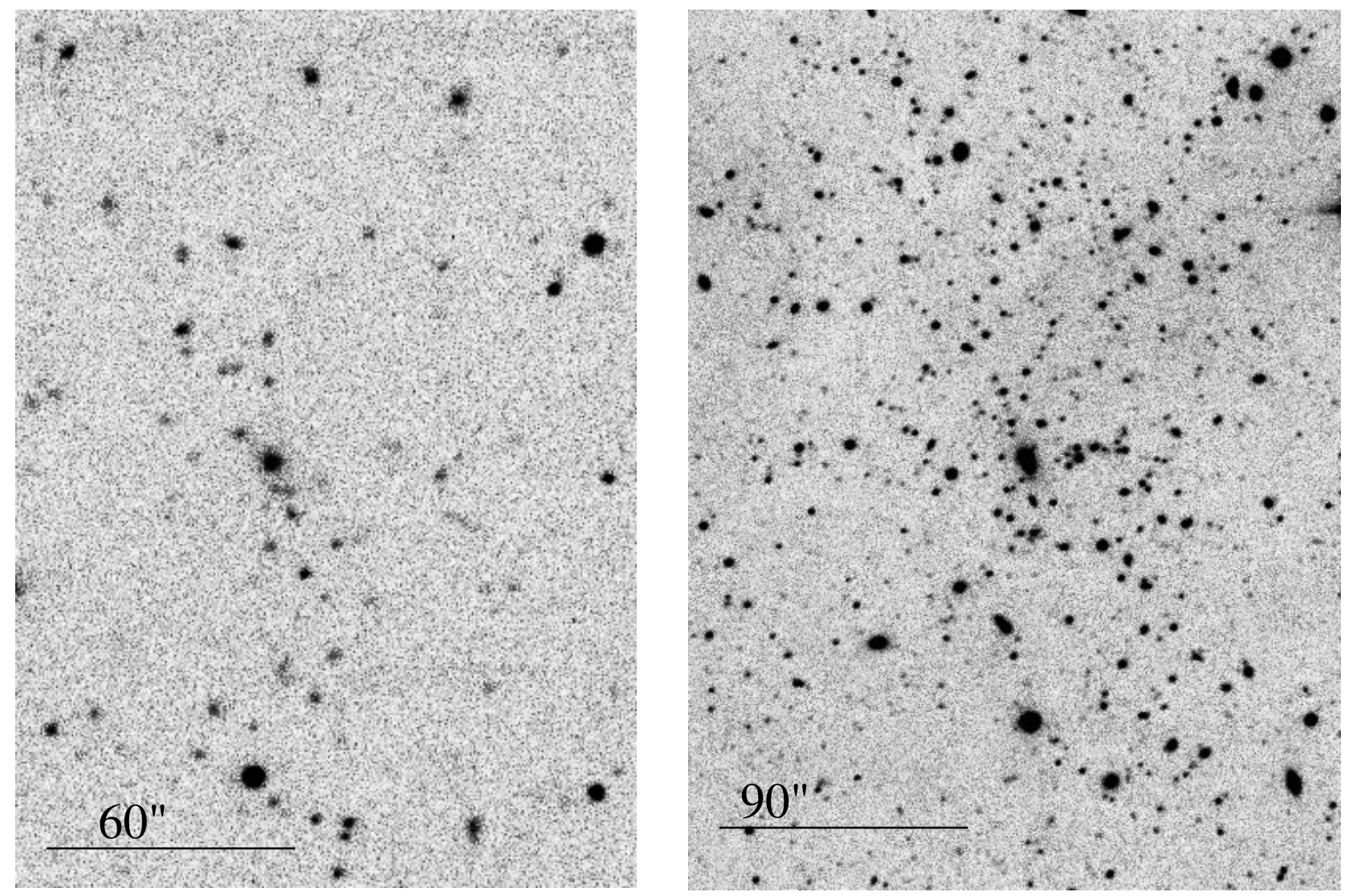

Fig. 4. $R$ band images of cluster V05 (left panel) and cluster V10 (right panel). ETS reveal clusters at $z=0.7$ and $z=0.6$, respectively. The central galaxies in these images correspond to brighter galaxies in the ETS of these systems. North is up and East is left.

We now turn to P33, P36 and P63 that are in the PDCS regions not covered by our run of the VGCF. The photometric and spectroscopic data we have for these clusters provide a further test of our ability to identify possible ETS and to estimate the photometric redshift of the cluster.

First of all we present in Fig. 5 the CMD of these clusters together with the ETS we identify following the same procedure as in the previous section. The black circles are the galaxies we use in the fit of the color-magnitude relation with fixed slope (see Sect. 5). Crosses are galaxies with spectroscopic redshift: we use all these galaxies in the fit, but one: the reddest galaxy in P33.

The photometric redshifts we derive for P33, P36 and P63 are $z_{\text {phot }}=0.64 \pm 0.04,0.18 \pm 0.02$ and $0.56 \pm 0.06$, respectively.

Cluster P33 presents a broad peak of 10 galaxies in redshift space. The peak is at a mean redshift $z=0.640 \pm 0.004$, the same redshift we estimate from the ETS. The velocity dispersion we compute is $\sigma_{\mathrm{v}}=2214_{-662}^{+1133} \mathrm{~km} \mathrm{~s}^{-1}$ in the cluster rest frame. This is an unrealistic velocity dispersion that could be caused by a too sparse sampling of the velocity field of the cluster. The redshift distribution is likely to be double-peaked. If we split the redshift distribution into two components at $z=0.6290$ and $z=0.6482$, we obtain two reasonable velocity dispersions $\sigma_{\mathrm{v}}=569 \mathrm{~km} \mathrm{~s}^{-1}$ and $\sigma_{\mathrm{v}}=389 \mathrm{~km} \mathrm{~s}^{-1}$. We note that Holden et al. (1997) find X-ray emission at the position of P33 in the ROSAT All Sky Survey (Snowden \& Schmitt 1990). This X-ray emission could be explained by a merging scenario between the two clumps (see e.g. Barrena et al. 2002 for a possible similar scenario in 1E0657-56 cluster of galaxies).

For clusters P36 and P63 we measure redshifts from long slit spectra: three redshifts for P36 and two for P63. The three galaxies of cluster P36 have redshifts close to $z=0.248$ and both galaxies of P63 are at $z=0.686$. Table 6 lists the redshifts of these cluster members. Again, the spectroscopic redshifts are quite close to the photometric estimates based on the ETS: $\left(z_{\text {phot }}-z\right)=0.07$ and 0.13 , respectively.

Redshifts we retrieve from the Sloan Digital Sky Survey team (SDSS; see, e.g., York et al. 2000) provide further evidence that the ETS is a reasonable indicator of the distance of our clusters. We find redshifts of 4 galaxies, one for each of the following clusters with ETS: V17, V23, V30 and V48.

Although one galaxy is not enough for a reliable redshift assignment, our galaxies with redshift have a rather large probability of being cluster members since they are among the brightest galaxies among 2D members and lie at the center of the angular distribution of counts. We list the redshifts in Table 7. For 3 out of 4 clusters with ETS we find $z_{\text {phot }}-z<0.02$. Cluster V17 has $z_{\text {phot }}-z=0.08$.

Finally, we consider the spectroscopic redshifts of Holden et al. (1999) for clusters P11, P12 and P23. Holden et al. (1999) estimate for these 3 clusters $z=0.327 \pm 0.003, z=0.263 \pm 0.002$ and $z=0.129 \pm 0.001$, respectively. We compare these values with our photometric redshift estimates: $z=0.36,0.32$ 
Table 6. Redshifts of member galaxies.

\begin{tabular}{|c|c|c|c|c|}
\hline ID & $\begin{array}{c}\mathrm{RA}_{2000} \\
\text { (hh:mm:ss) }\end{array}$ & $\begin{array}{c}\operatorname{Dec}_{2000} \\
\left({ }^{\circ}:^{\prime}:^{\prime \prime}\right)\end{array}$ & $z$ & $\delta_{z}$ \\
\hline \multicolumn{5}{|c|}{ Cluster V04 (P04) } \\
\hline $1^{(+)}$ & $00: 29: 13.1$ & $05: 09: 15$ & 0.5492 & 0.0004 \\
\hline $2^{(+)}$ & $00: 29: 12.1$ & 05:08:32 & 0.5512 & 0.0003 \\
\hline $3^{(+)}$ & $00: 29: 11.0$ & 05:09:27 & 0.5509 & 0.0004 \\
\hline $4^{(+)}$ & $00: 29: 10.1$ & 05:09:10 & 0.5494 & 0.0003 \\
\hline $5^{(+)}$ & $00: 29: 15.6$ & 05:07:11 & 0.5539 & 0.0002 \\
\hline $6^{(+)}$ & $00: 29: 14.4$ & 05:07:58 & 0.5504 & 0.0001 \\
\hline $7^{(+)}$ & 00:29:11.0 & 05:08:45 & 0.5513 & 0.0001 \\
\hline $8^{(+)}$ & $00: 29: 15.9$ & 05:10:03 & 0.5481 & 0.0001 \\
\hline $9^{(+)}$ & 00:29:17.7 & 05:10:00 & 0.5498 & 0.0002 \\
\hline \multicolumn{5}{|c|}{ Cluster V06 } \\
\hline 1 & $00: 29: 13.7$ & $05: 17: 04$ & 0.3678 & 0.0007 \\
\hline 2 & $00: 29: 16.0$ & $05: 17: 45$ & 0.3652 & 0.0002 \\
\hline 3 & $00: 29: 16.1$ & $05: 17: 56$ & 0.3640 & 0.0003 \\
\hline 4 & $00: 29: 18.6$ & 05:19:01 & 0.3671 & 0.0004 \\
\hline 5 & 00:29:09.6 & $05: 13: 50$ & 0.3691 & 0.0002 \\
\hline 6 & $00: 29: 15.9$ & $05: 17: 49$ & 0.3682 & 0.0006 \\
\hline 7 & $00: 29: 25.6$ & 05:17:06 & 0.3619 & 0.0002 \\
\hline 8 & $00: 29: 15.8$ & $05: 17: 37$ & 0.3658 & 0.0001 \\
\hline 9 & $00: 29: 23.7$ & $05: 20: 33$ & 0.3620 & 0.0001 \\
\hline \multicolumn{5}{|c|}{ Cluster P33 } \\
\hline $1^{(*)}$ & $09: 52: 11.7$ & $47: 16: 21$ & 0.6259 & 0.0001 \\
\hline $2^{(*)}$ & $09: 52: 12.8$ & $47: 16: 39$ & 0.6470 & 0.0004 \\
\hline $3^{(*)}$ & $09: 52: 14.2$ & $47: 17: 12$ & 0.6492 & 0.0004 \\
\hline $4^{(+)}$ & $09: 52: 12.9$ & $47: 17: 29$ & 0.6240 & 0.0001 \\
\hline $5^{(+)}$ & 09:52:05.6 & $47: 15: 50$ & 0.6356 & 0.0003 \\
\hline $6^{(+)}$ & $09: 52: 18.3$ & $47: 14: 26$ & 0.6559 & 0.0005 \\
\hline $7^{(+)}$ & 09:52:00.7 & $47: 16: 12$ & 0.6502 & 0.0004 \\
\hline $8^{(+)}$ & 09:51:49.2 & $47: 14: 18$ & 0.6312 & 0.0002 \\
\hline $9^{(+)}$ & 09:51:40.1 & 47:14:09 & 0.6307 & 0.0002 \\
\hline $10^{(+)}$ & 09:52:50.6 & $47: 13: 37$ & 0.6478 & 0.0001 \\
\hline \multicolumn{5}{|c|}{ Cluster P36 } \\
\hline 1 & 09:53:53.9 & $47: 40: 11$ & 0.2459 & 0.0002 \\
\hline 2 & $09: 53: 54.0$ & $47: 40: 24$ & 0.2502 & 0.0001 \\
\hline 3 & 09:53:54.2 & $47: 40: 36$ & 0.2484 & 0.0002 \\
\hline \multicolumn{5}{|c|}{ Cluster P63 } \\
\hline 1 & $13: 24: 20.8$ & $30: 12: 42$ & 0.6870 & 0.0003 \\
\hline 2 & $13: 24: 21.5$ & 30:13:01 & 0.6866 & 0.0009 \\
\hline
\end{tabular}

(*) Two redshifts: from our TNG observations and from CFHT archival data. The discrepancy between measurements is less than $50 \mathrm{~km} \mathrm{~s}^{-1}$. ${ }^{(+)}$Redshift from CFHT archival data.

and 0.19 . The difference between photometric and spectroscopic redshifts is $z_{\text {phot }}-z<0.06$.

Thus, we find that spectroscopic and photometric redshifts are in good agreement. Table 9 summarizes the results obtained with both techniques. The average difference between photometric and spectroscopic redshifts is $\left\langle z_{\text {phot }}-z_{\text {spec }}\right\rangle=0.05 \pm 0.04$, consistent with our estimate of external errors $\delta z \simeq 0.1$ (see Sect. 5).

\section{Discussion of the VGCF and PDCS cluster detections}

The basic characteristics of the VGCF and matched-filter algorithm (PLG96) are the numbers of clusters they detect. Table 8 summarizes the situation. We identify a total of 48 clusters, including 3 possible substructures. 25 of the 48 clusters are particularly reliable since we identify an ETS in their CMD. Given that several clusters are rather poor, the lack of an ETS is not to be taken as a proof that the cluster is a chance projection of unrelated galaxies.

Within the PDCS borders we identify 41 clusters, i.e. 13 more clusters than PLG96 (all the 25 reliable clusters are among these 41 clusters). Because only a fraction of our 41 clusters coincide with PDCS clusters, the difference between the numbers of VGCF and PDCS clusters does not reflect the actual performances of the two algorithms. A more detailed discussion is needed in order to understand how complete the VGCF and PDCS catalogs are.

We start from Fig. 1. As already described in Sect. 4, in Fig. 1 our clusters are the solid thick circles, PDCS clusters are the long-dashed circles marked with a $\mathrm{P}$ followed by the PDCS identification number. For an easier comparison, in what follows, we consider only VGCF clusters that fall within the PDCS0 and PDCS2 areas.

There are 20 clusters that are identified by both the VGCF and the matched-filter (PLG96). These "common" clusters have properties that are representative of their parent samples in these fields.

We then identify 21 clusters that PLG96 miss. These clusters fall into three categories: a) clusters that are identified within larger clusters, b) clusters that are very close to other (larger) clusters, and c) apparently "normal" isolated clusters.

Clusters V12, V14, and V22 are detected within clusters V13, V33, and V21 respectively. These clusters could have not been detected by the matched-filter algorithm. These (poor) clusters could be physical substructures or projected background clusters. We find no ETS in their CMD. The same is true for V22 that lies inside PDCS2 but is included within V21 identified outside PDCS2.

The category of small clusters identified close to larger clusters includes 6 systems (V11, V15, V16, V17, V24, V41). In detail, we consider as "close" those clusters with centers at a distance $D_{12}$ such that $\max \left(R_{1}, R_{2}\right)<D_{12}<R_{1}+R_{2}$, where $R_{1}$ and $R_{2}$ are the radii of the two clusters.

Clusters in this category mark a significant difference between VGCF and matched-filter techniques. For 4 out of 6 clusters we identify a sequence of early-type galaxies that make these detections particularly reliable. The origin of this difference is likely to be the smoothing performed by the matched filter algorithm. The relatively large smoothing length used for the detection of rich and/or low-redshift clusters does not allow the detection of small neighboring structures.

Finally, we identify 12 well-isolated clusters missing in the PDCS catalog (V05, V06, V16, V18, V20, V31, V34, V37, V39,V44, V45, V47). No obvious property of these clusters explains their absence in the PDCS catalog, except (possibly) for cluster V30 that is only partially contained within the PDCS field.

We find an ETS in the CMD of 6 out of the 12 wellisolated clusters. Spectroscopic observations confirm cluster V06 (among the 6 with ETS) and add V04 (no ETS) to the list of confirmed clusters. The relatively large fraction of 

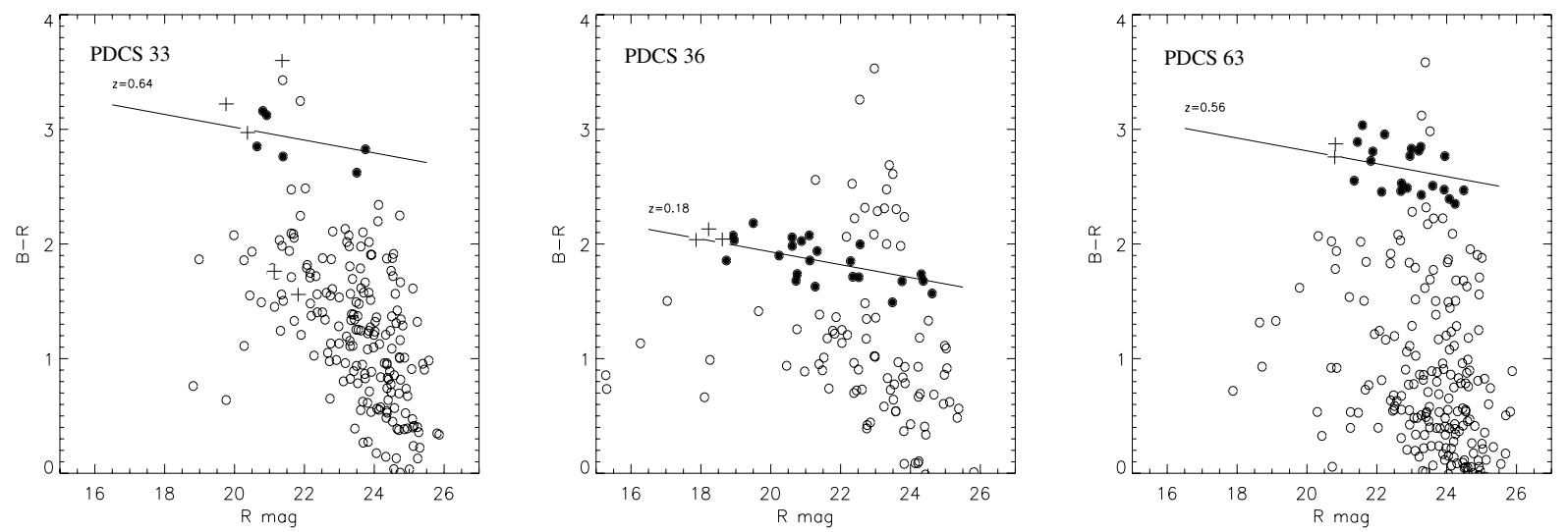

Fig. 5. $(B-R, R)$ CMD of clusters $\mathrm{P} 33, \mathrm{P} 36$ and $\mathrm{P} 63$. The ETS are linear fits with constant slopes to black filled circles and crosses. Crosses are cluster members with spectroscopic redshift.

Table 7. Redshifts obtained from SDSS.

\begin{tabular}{ccccc}
\hline \hline Cluster & $\begin{array}{c}\alpha_{2000} \\
(\mathrm{hh}: \mathrm{mm}: \mathrm{ss})\end{array}$ & $\begin{array}{c}\delta_{2000} \\
\left({ }^{\circ}:^{\prime}:{ }^{\prime \prime}\right)\end{array}$ & $z$ & $\delta z$ \\
\hline Galaxy in V17 & $02: 27: 45$ & $00: 59: 20$ & 0.3965 & 0.0002 \\
Galaxy in V23 & $02: 28: 33$ & $00: 57: 18$ & 0.3371 & 0.0002 \\
Galaxy in V30 & $02: 26: 24$ & $01: 00: 40$ & 0.3979 & 0.0002 \\
Galaxy in V48 & $02: 25: 23$ & $01: 08: 50$ & 0.2674 & 0.0002 \\
\hline
\end{tabular}

Table 8. Cluster counts.

\begin{tabular}{cccc}
\hline \hline & Confirmed & Not confirmed & Total \\
\hline & Within the total $B R+V I$ area \\
VGCF & 25 & 23 & 48 \\
\hline \multicolumn{4}{c}{ Within PDCS0 and PDCS2 } \\
VGCF & 25 & 15 & 41 \\
PLG96 & - & 28 & 28 \\
\hline
\end{tabular}

"color confirmations" and the two spectroscopic confirmations lead us to the conclusion that VGCF clusters significantly contribute to the completeness of cluster samples identified with a matched-filter algorithm.

PLG96 identify 8 clusters that the VGCF misses. Of these 8 clusters, only 2 are in PDCSO. All other clusters are in PDCS2. All but one of these clusters are located in the right ascension range $2^{\mathrm{h}} 26^{\mathrm{m}}<\alpha<2^{\mathrm{h}} 31^{\mathrm{m}}$, less than $1 / 3$ of the total area of the PDCS2 field. The clusters with $2^{\mathrm{h}} 26^{\mathrm{m}}<\alpha<2^{\mathrm{h}} 31^{\mathrm{m}}$ are P15, P17, P25, P26, P27. We identify clusters P26 and P27 only in the $V$ band because we have no $I$-band data at their position. These clusters do not enter our catalog since we require the detection in two bands for a cluster. We identify P15, P17 and P25 only in the $V$ band too, even if we have $I$-band data at their position. We also note that $\mathrm{P} 15$ and $\mathrm{P} 27$ have the smallest radii among the PDCS clusters in PDCS0 and PDCS2.

Thus, there are 46 clusters identified by us and/or PLG96 within the two PDCS fields (excluding the 3 possible "substructures" V12, V14 and V22). The total number of clusters is larger than the number of VGCF clusters identified within the same region by $20 \%$ and larger than the number of PDCS clusters by more than $60 \%$.

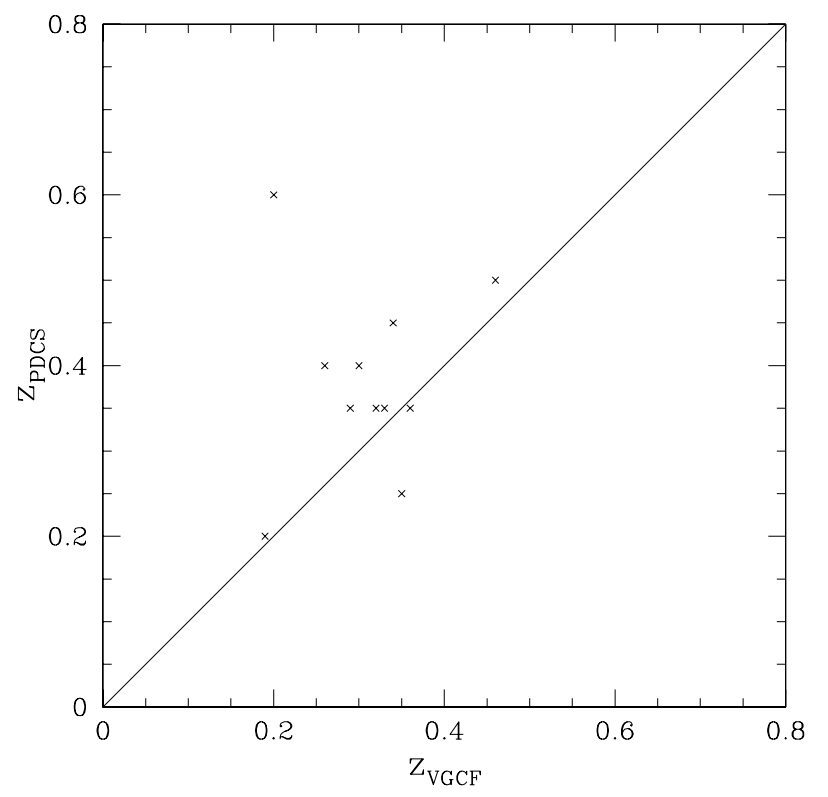

Fig. 6. Comparison between VGCF and PDCS redshift estimates. The diagonal line illustrates a one-to-one relation and it is not a fit.

The comparison between our catalog and the PDCS catalog can be extended to redshift estimates for 11 clusters with redshifts estimated by both PLG96 and us. We plot these systems in Fig. 6. In the same figure we draw a diagonal line representing a one-to-one relation. For all but one system $(\mathrm{V} 03=\mathrm{P} 01)$ the redshift estimates agree well within the specified uncertainties, $\delta z=0.1$ for both us and PLG96.

\section{Summary}

Our aim is to test the performances of the VGCF on deep $B$, $R$ wide field images of two PDCS fields (PDCSO at $0^{\mathrm{h}}$ and PDCS2 at $2^{\mathrm{h}}$ ). In a previous paper (R01) we ran the VGCF on PDCS catalogs of galaxies in the $V$ and $I$ bands (PLG96). Here we investigate the merits of the VGCF and the PDCS catalogs (obtained with a matched filter algorithm) in two further PDCS fields. In these fields we also have deep $B$ and $R$ wide field images at our disposal. By comparing the PDCS and VGCF catalogs, we evaluate the relative incompleteness of the 
Table 9. Clusters with both photometric and spectroscopic redshifts.

\begin{tabular}{cccc}
\hline \hline Cluster & PDCS & $\bar{z}_{\text {phot }}$ & \multicolumn{1}{c}{$z_{\text {spec }}$} \\
\hline V06 & - & 0.43 & $0.3658 \pm 0.0009^{(1)}$ \\
V13 & P12 & 0.32 & $0.2630 \pm 0.0020^{(1)}$ \\
V17 & - & 0.32 & $0.3965 \pm 0.0002^{(2)}$ \\
V19 & P23 & 0.19 & $0.1290 \pm 0.0010^{(1)}$ \\
V23 & P20 & 0.34 & $0.3371 \pm 0.0002^{(2)}$ \\
V30 & - & 0.38 & $0.3979 \pm 0.0002^{(2)}$ \\
V36 & P11 & 0.36 & $0.3270 \pm 0.0030^{(1)}$ \\
V48 & P24 & 0.26 & $0.2674 \pm 0.0002^{(2)}$ \\
- & P33 & 0.64 & $0.6400 \pm 0.0040$ \\
- & P36 & 0.18 & $0.2480 \pm 0.0020$ \\
- & P63 & 0.56 & $0.6863 \pm 0.0003$ \\
\hline
\end{tabular}

(1) From Holden et al. (1999).

(2) Redshift from SDSS data for a single galaxy that falls into the brightest part of the ETS of the cluster.

two catalogs. This information is relevant to the cosmological abundance of clusters and to the evaluation of selection biases of optical cluster samples.

We run the VGCF in magnitude bins on our BVRI catalogs and obtain a final list of 48 clusters. Of these clusters, 3 are identified within other clusters and could be either physical substructures or projected background clusters.

We use our BVRI photometry to find evidence that our clusters are real physical systems. We analyze the CMD of each cluster, and search for the presence of an ETS. We consider these clusters as reliable VGCF identifications and include them in the list of "confirmed clusters"

Once we identify an ETS, we estimate the photometric redshift of the cluster by comparing the observed ETS to a grid of ETS obtained by redshifting the ETS of Coma (for the $B R$ CMD) and A118 (for the VI CMD).

We find an ETS in both the $(B-R, R)$ and $(V-I, I)$ CMD of 7 clusters. There are 18 further clusters that have a recognizable ETS in one of the two CMD. Redshift estimates range from $z_{\mathrm{ETS}}=0.12(\mathrm{~V} 31)$ to $z_{\mathrm{ETS}}=0.75$ (V5) with an uncertainty of the order of $\delta z=0.1$.

For three of our VGCF clusters (V04, V06, V18) we obtain additional spectroscopic data with our own observations with the DOLoRes spectrograph at the TNG or from public databases. We confirm V04 and V06 as real physical systems. Consistent with our expectations, V18 that appears to be a projection of physically unrelated galaxies has no detectable ETS.

We then compare our cluster catalog with the PDCS cata$\log$ (PLG96). There are 41 VGCF clusters in the PDCS0 and PDCS2 areas where PLG96 identify 28 clusters.

A total of 20 clusters are identified by both the VGCF and the matched-filter algorithm of PLG96.

We identify 21 clusters that PLG96 miss. These clusters fall into three categories: a) 3 clusters that are identified within larger clusters, b) 6 clusters that are very close to other (larger) clusters, and c) 12 apparently "normal" isolated clusters. In particular case b) clusters indicate the main draw-back of the smoothing required by the matched-filter algorithm.

PLG96 identify 8 clusters that the VGCF misses.
The VGCF identifies a large fraction of the PDCS clusters $(\sim 70 \%)$. For part of the PDCS fields we only have $V$ and $I$ data. If we relax our criterion for cluster identification in these areas and require only a detection in the $V$ band, the VGCF identifies $\sim 90 \%$ of the PDCS clusters.

Adding all the independent cluster identifications, the total number of clusters within PDCS0 and PDCS2 is 46. This number is $\sim 20 \%$ larger than the number of clusters identified with the VGCF and $\sim 60 \%$ larger than the number of PDCS clusters.

These results confirm a) that the VGCF is a competitive algorithm for the identification of clusters, b) that a combined catalog of matched-filter and VGCF clusters constitutes a significant progress toward a more complete selection of clusters from bidimensional optical data.

Acknowledgements. We wish to thank Marc Postman, who kindly provided $V$ and $I$ catalogs of the $0^{\mathrm{h}}$ and $2^{\mathrm{h}}$ PDCS fields and Alex Oscoz for his help during the observations at the INT and useful comments and suggestions.

This publication is based on observations made on the island of La Palma with the Isaac Newton Telescope (INT) and Jacobus Kaptein Telescope (JKT) both operated by the Isaac Newton Group, with the Italian Telescopio Nazionale Galileo (TNG) operated by the Centro Galileo Galilei dell'INAF (Istituto Nazionale di Astrofisica), and the Nordic Optical Telescope (NOT) in the Spanish Observatorio del Roque de los Muchachos of the Instituto de Astrofisica de Canarias. This publication also makes use of data from the Sloan Digital Sky Survey (SDSS) public archive. Funding for the creation and distribution of the SDSS Archive has been provided by the Alfred P. Sloan Foundation, the Participating Institutions, the National Aeronautics and Space Administration, the National Science Foundation, the US Department of Energy, the Japanese Monbukagakusho, and the Max Planck Society. The SDSS is managed by the Astrophysical Research Consortium (ARC) for the Participating Institutions. The Participating Institutions are The University of Chicago, Fermilab, the Institute for Advanced Study, the Japan Participation Group, The Johns Hopkins University, the Korean Scientist Group, Los Alamos National Laboratory, the Max-Planck-Institute for Astronomy (MPIA), the Max-Planck-Institute for Astrophysics (MPA), New Mexico State University, University of Pittsburgh, Princeton University, the United States Naval Observatory and the University of Washington.

\section{References}

Adami, C., Holden, B. P., Castander, F. J., et al. 2000, AJ, 120, 1 Bahcall, N. A., McKay, T. A., Annis, J., et al. 2003, ApJS, 148, 243

Barrena, R., Biviano, A., Ramella, M., et al. 2002, A\&A, 386, 816

Beers, T. C., Kage, J. A., Preston, G. W., \& Shectman, S. A. 1990, AJ, 100, 849

Bertin, E., \& Arnouts, S. 1996, A\&AS, 117, 393

Boschin, W. 2002, A\&A, 396, 397

Burstein, D., \& Heiles, C. 1982, AJ, 87, 1165

Busarello, G., Merluzzi, P., La Barbera, F., et al. 2002, A\&A, 389, 787

Cousins, A. W. J. 1976, MemRAS, 81, 25

Dalton, G. B., Maddox, S. J., Sutherland, W. J., \& Efstathiou, G. 1997, MNRAS, 289, 263

Donahue, M., Mack, J., Scharf, C., et al. 2001, ApJ, 552, L93

Gilbank, D. G., Bower, R. G., Castander, F. J., \& Ziegler, B. L. 2004, MNRAS, 348, 551

Gladders, M. D., \& Yee, H. K. C. 2000, AJ, 120, 2148

Gladders M. D., \& Yee, H. K. C. 2005, ApJS, 157, 1 
Gonzalez, A. H., Zaritsky, D., Dalcanton, J. J., \& Nelson, A. 2001, ApJS, 137, 117

Goto, T., Sekiguchi, M., Nichol, R. C., et al. 2002, AJ, 123, 1807

Gullixson, C. A. 1992, ASP Conf. Ser., 23, 130

Harrison, E. R., \& Noonan, T. W. 1979, ApJ, 232, 18

Holden, B. P., Romer, A. K., Nichol, R. C., \& Ulmer, M. P. 1997, AJ, 114,170

Holden, B. P., Nichol, R. C., Romer, A. K., et al. 1999, AJ, 118, 2002

Johnson, H. L., \& Morgan, W. W. 1953, ApJ, 117, 313

Kiang, T. 1966, Z. Astrophys., 64, 433

Kinney, A. L., Calzetti, D., Bohlin, R. C., et al. 1996, ApJ, 467, 38

Kim, R., Seung, J., \& Kepner, J. V. 2002, AJ, 123, 20

Lopes, P. A. A., de Carvalho, R. R., Gal, R. R., et al. 2004, AJ, 128, 1017

Lumsden, S. L., Nichol, R. C., Collins, C. A., \& Guzzo, L. 1992, MNRAS, 258, 1

Moretti, A., Guzzo, L., Campana, S., et al. 2004, A\&A, 428, 21

Nonino, M., Bertin, E., da Costa, L., et al. 1999, A\&AS, 137, 51

Olsen, L. F., Scodeggio, M., da Costa, L., et al. 1999, A\&A, 345, 681
Phelps, R. L. 1997, ApJ, 483, 826

Poggianti, B. M. 1997, A\&AS, 122, 399

Postman, M., Lubin, L. M., Gunn, J. E., et al. 1996, A\&AS, 137, 83 (PLG96)

Postman, M., Lauer, T. R., Oegerle, W., \& Donahue, M. 2002, ApJ, 579,93

Ramella, M., Boschin, W., Fadda, D., \& Nonino, M. 2001, A\&A, 368, 776 (R01)

Rosati, P., della Ceca, R., Norman, C., \& Giacconi, R. 1998, A\&A, 331,41

Secker, J., Harris, W. E., \& Plummer, J. D. 1997, PASP, 109, 1377

Snowden, S. L., \& Schmitt, J. H. M. M. 1990, ApSS, 171, 207

Tonry, J., \& Davis, M. 1979, AJ, 84, 1511

Willick, J. A., Thompson, K. L., Mathiesen, B. F., et al. 2001, PASP, 113,658

York, D. G., Adelman, J., Anderson, J. E., et al. 2000, AJ, 120, 1579

Zaritsky, D., Nelson, A. E., Dalcanton, J. J., \& González, A. H. 1997, ApJ, 480, L91 
R. Barrena et al.: VGCF detection of galaxy systems at intermediate redshifts, Online Material p 1

\section{Online Material}


Table 5. Cluster catalog.

\begin{tabular}{|c|c|c|c|c|c|c|c|c|c|c|}
\hline ID & $C_{B}$ & $C_{V}$ & $C_{R}$ & $\overline{C_{I}}$ & $\begin{array}{c}\alpha_{2000} \\
\text { (hh:mm:ss) }\end{array}$ & $\begin{array}{c}\delta_{2000} \\
\left({ }^{\circ}:^{\prime}:{ }^{\prime \prime}\right)\end{array}$ & $\begin{array}{c}\text { Radius } \\
\left({ }^{\prime \prime}\right)\end{array}$ & $\overline{\bar{z}_{\text {phot }}}$ & ZPDCS & Remarks $^{a}$ \\
\hline \multicolumn{11}{|c|}{ Field F0028+0515 } \\
\hline V01 & 0 & 6.79 & 5.35 & 0 & $00: 28: 31.7$ & $05: 18: 18$ & 97 & - & 0.6 & $\mathrm{~A}, \mathrm{P} 08$ \\
\hline V02 & 5.42 & 5.13 & 0 & 0 & $00: 28: 37.0$ & 05:07:48 & 119 & - & 0.6 & $\mathrm{~A}, \mathrm{P} 03$ \\
\hline V03 & 0 & 5.73 & 0 & 6.41 & $00: 29: 02.9$ & 05:01:30 & 113 & 0.20 & 0.6 & $\mathrm{~A}, \mathrm{P} 01$ \\
\hline V04 & 5.92 & 7.78 & 10.08 & 8.43 & $00: 29: 13.0$ & 05:08:13 & 155 & - & 0.5 & $\mathrm{~A}, \mathrm{P} 04\left(z_{\mathrm{spec}}=0.550\right)$ \\
\hline V05 & 5.67 & 5.29 & 0 & 6.06 & $00: 29: 13.7$ & $05: 26: 56$ & 108 & 0.75 & - & A \\
\hline V06 & 0 & 5.00 & 4.47 & 5.66 & $00: 29: 16.3$ & $05: 18: 14$ & 94 & 0.43 & - & $\mathrm{A},\left(z_{\mathrm{spec}}=0.366\right)$ \\
\hline V07 & 5.72 & 5.16 & 0 & 5.68 & $00: 29: 31.2$ & $05: 03: 40$ & 115 & 0.30 & 0.4 & $\mathrm{~A}, \mathrm{P} 02$ \\
\hline V08 & 6.96 & 5.50 & 4.95 & 7.42 & 00:29:51.6 & 05:12:29 & 158 & - & 0.4 & A, P06 \\
\hline \multicolumn{11}{|c|}{ Field F0027+0555 } \\
\hline V09 & 8.00 & - & 7.12 & - & $00: 26: 29.5$ & $05: 41: 17$ & 97 & - & - & B \\
\hline V10 & 11.68 & - & 10.58 & - & $00: 26: 41.3$ & $05: 47: 38$ & 90 & 0.61 & - & B \\
\hline V11 & 7.00 & 0 & 5.67 & 0 & $00: 27: 30.5$ & $05: 56: 46$ & 68 & - & - & A \\
\hline V12 & 0 & 5.66 & 5.58 & 0 & $00: 27: 55.0$ & 05:59:20 & 104 & - & - & A, inside V13 \\
\hline V13 & 11.26 & 10.03 & 9.77 & 9.81 & $00: 27: 53.5$ & 05:57:11 & 284 & 0.32 & 0.35 & $\mathrm{~A}, \mathrm{P} 12$ \\
\hline \multicolumn{11}{|c|}{ Field F0228+0115 } \\
\hline V14 & 5.82 & 0 & 6.35 & 0 & $02: 26: 56.4$ & 01:03:14 & 101 & - & - & $\mathrm{A}$, inside V33 \\
\hline V15 & 0 & 5.06 & 6.00 & 0 & $02: 27: 08.9$ & $01: 03: 25$ & 122 & - & - & A \\
\hline V16 & 7.42 & 7.00 & 0 & 4.62 & $02: 27: 20.9$ & 01:09:18 & 119 & 0.31 & - & A \\
\hline V17 & - & 8.50 & 9.35 & 7.22 & $02: 27: 42.2$ & $00: 59: 24$ & 148 & 0.32 & - & A \\
\hline V18 & 5.66 & 8.94 & 8.92 & 8.14 & $02: 27: 48.0$ & $01: 13: 44$ & 194 & - & - & A \\
\hline V19 & 5.00 & 6.94 & 6.93 & 7.60 & 02:27:53.0 & 01:04:52 & 191 & 0.19 & 0.2 & $\mathrm{~A}, \mathrm{P} 23$ \\
\hline V20 & 6.00 & 4.47 & 5.20 & 0 & $02: 28: 15.6$ & $01: 13: 55$ & 83 & 0.47 & - & A \\
\hline V21 & 8.05 & - & 11.01 & - & $02: 28: 23.5$ & 01:30:40 & 202 & 0.44 & - & $\mathrm{B}$ \\
\hline V22 & 8.00 & - & 8.08 & - & $02: 28: 27.1$ & $01: 27: 54$ & 65 & - & - & $\mathrm{A}$, inside $\mathrm{V} 21$ \\
\hline $\mathrm{V} 23$ & - & 6.36 & 7.00 & 7.00 & $02: 28: 32.4$ & $00: 57: 18$ & 101 & 0.34 & 0.45 & $\mathrm{~A}, \mathrm{P} 20$ \\
\hline V24 & 5.20 & - & 5.00 & - & $02: 28: 43.4$ & 01:28:05 & 97 & 0.28 & - & A \\
\hline V25 & 9.26 & 6.00 & 6.68 & - & $02: 28: 49.7$ & $01: 23: 13$ & 288 & - & 0.2 & A, $\mathrm{P} 28$ \\
\hline \multicolumn{11}{|c|}{ Field F0226+0026 } \\
\hline V26 & 5.82 & - & 4.95 & - & $02: 25: 19.0$ & $00: 49: 37$ & 104 & - & - & B \\
\hline V27 & 6.20 & - & 7.89 & - & $02: 25: 43.7$ & $00: 49: 55$ & 104 & - & - & B \\
\hline V28 & 6.38 & - & 6.32 & - & $02: 25: 47.3$ & 01:07:05 & 108 & - & - & B \\
\hline V29 & 6.10 & - & 12.49 & - & $02: 26: 06.5$ & 01:11:02 & 223 & - & - & B \\
\hline V30 & 7.57 & 8.96 & 18.01 & 6.93 & $02: 26: 25.7$ & 01:00:40 & 248 & 0.38 & - & A \\
\hline V31 & 0 & 0 & 5.31 & 6.93 & $02: 26: 35.3$ & $01: 12: 58$ & 86 & 0.12 & - & A \\
\hline V32 & 5.37 & 6.38 & 4.91 & 6.06 & $02: 26: 39.6$ & $00: 57: 50$ & 133 & - & 0.35 & $\mathrm{~A}, \mathrm{P} 21$ \\
\hline V33 & 4.44 & 7.84 & 8.23 & 8.27 & 02:26:53.3 & 01:06:29 & 234 & - & 0.5 & $\mathrm{~A}, \mathrm{P} 22$ \\
\hline \multicolumn{11}{|c|}{ PDCS 0 (no overlap with F0028+0515 or F0027+0555) } \\
\hline V34 & & 6.12 & & 6.72 & $00: 27: 29.3$ & 05:33:00 & 101 & 0.32 & - & $\mathrm{C}$ \\
\hline V35 & & 8.33 & & 6.83 & $00: 28: 55.2$ & 05:48:04 & 248 & 0.33 & 0.35 & $\mathrm{C}, \mathrm{P} 10$ \\
\hline V36 & & 8.00 & & 8.22 & $00: 29: 41.3$ & 05:50:35 & 245 & 0.36 & 0.35 & $\mathrm{C}, \mathrm{P} 11$ \\
\hline V37 & & 7.25 & & 6.76 & $00: 29: 48.5$ & 05:58:08 & 130 & - & - & $\mathrm{C}$ \\
\hline V38 & & 6.40 & & 6.03 & $00: 30: 47.8$ & $05: 37: 44$ & 144 & 0.29 & 0.35 & C, P09 \\
\hline V39 & & 6.00 & & 5.77 & $00: 30: 53.5$ & $05: 54: 58$ & 94 & 0.39 & - & $\mathrm{C}$ \\
\hline V40 & & 8.49 & & 5.30 & $00: 30: 55.2$ & $05: 14: 49$ & 227 & 0.35 & 0.25 & $\mathrm{C}, \mathrm{P} 07$ \\
\hline V41 & & 5.00 & & 6.00 & $00: 31: 16.1$ & $05: 16: 37$ & 104 & 0.28 & - & $\mathrm{C}$ \\
\hline \multicolumn{11}{|c|}{ PDCS 2 (no overlap with F0228+0115 or F0226+0026 fields) } \\
\hline V42 & & 7.00 & & 6.36 & $02: 27: 25.9$ & $00: 25: 19$ & 137 & - & 0.35 & $\mathrm{C}, \mathrm{P} 14$ \\
\hline V43 & & 6.93 & & 6.36 & $02: 27: 29.8$ & 00:39:07 & 112 & - & 0.4 & $\mathrm{C}, \mathrm{P} 18$ \\
\hline V44 & & 5.55 & & 5.13 & 02:28:01.9 & $00: 32: 35$ & 104 & - & - & $\mathrm{C}$ \\
\hline V45 & & 6.87 & & 5.73 & 02:28:02.4 & $00: 40: 52$ & 133 & 0.31 & - & $\mathrm{C}$ \\
\hline V46 & & 9.43 & & 11.35 & $02: 28: 27.8$ & $00: 31: 26$ & 126 & 0.46 & 0.5 & C, P16 \\
\hline V47 & & 5.33 & & 6.00 & 02:30:07.9 & $00: 46: 16$ & 104 & - & - & $\mathrm{C}$ \\
\hline V48 & & 8.55 & & 6.42 & 02:30:23.0 & 01:09:29 & 169 & 0.26 & 0.4 & $\mathrm{C}, \mathrm{P} 24$ \\
\hline
\end{tabular}

${ }^{a}$ In the column with remarks, an "A" corresponds to a cluster identified in an overlap region between our fields and PDCS0 or PDCS2, a "B" to a cluster identified in a region outside the PDCS fields, a "C" to a cluster identified in a PDCS region not overlapping our fields. PDCS counterparts are labeled with a "P" followed by the cluster ID in PLG96. We also add a remark indicating VGCF clusters that have their center within another VGCF cluster. 
R. Barrena et al.: VGCF detection of galaxy systems at intermediate redshifts, Online Material p 3
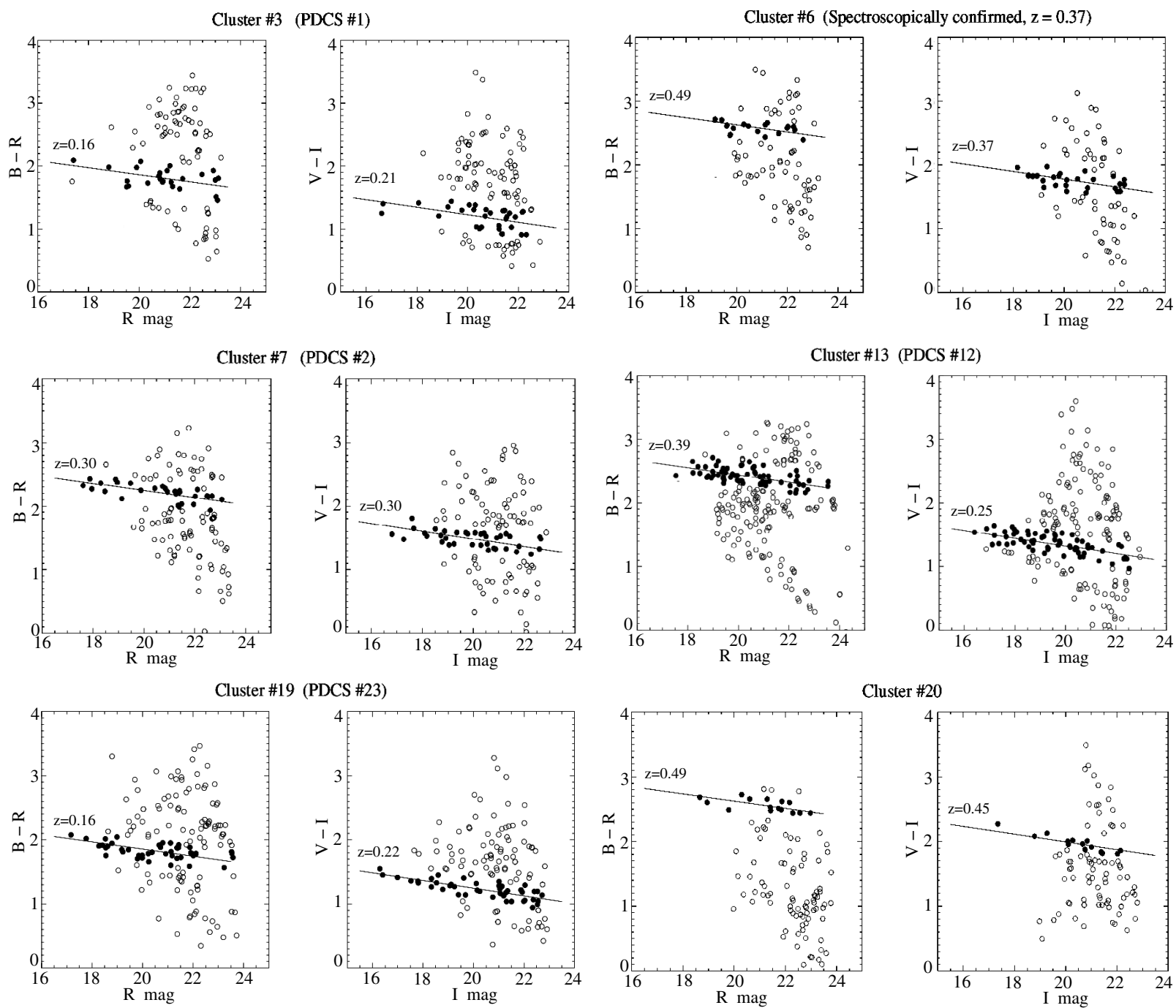

Cluster $\# 20$

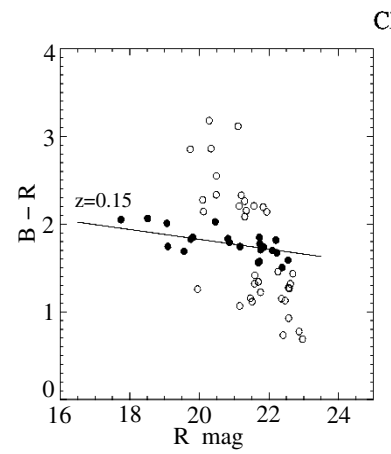

Cluster \#31

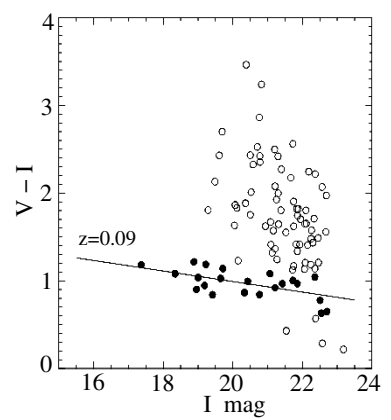

Fig. 2. $(B-R, R)$ and $(V-I, I)$ ETS and redshift estimates of clusters within our survey. This sample includes clusters with ETS in both $(B-R$, $R)$ and $(V-I, I)$ diagrams. 
R. Barrena et al.: VGCF detection of galaxy systems at intermediate redshifts, Online Material p 4
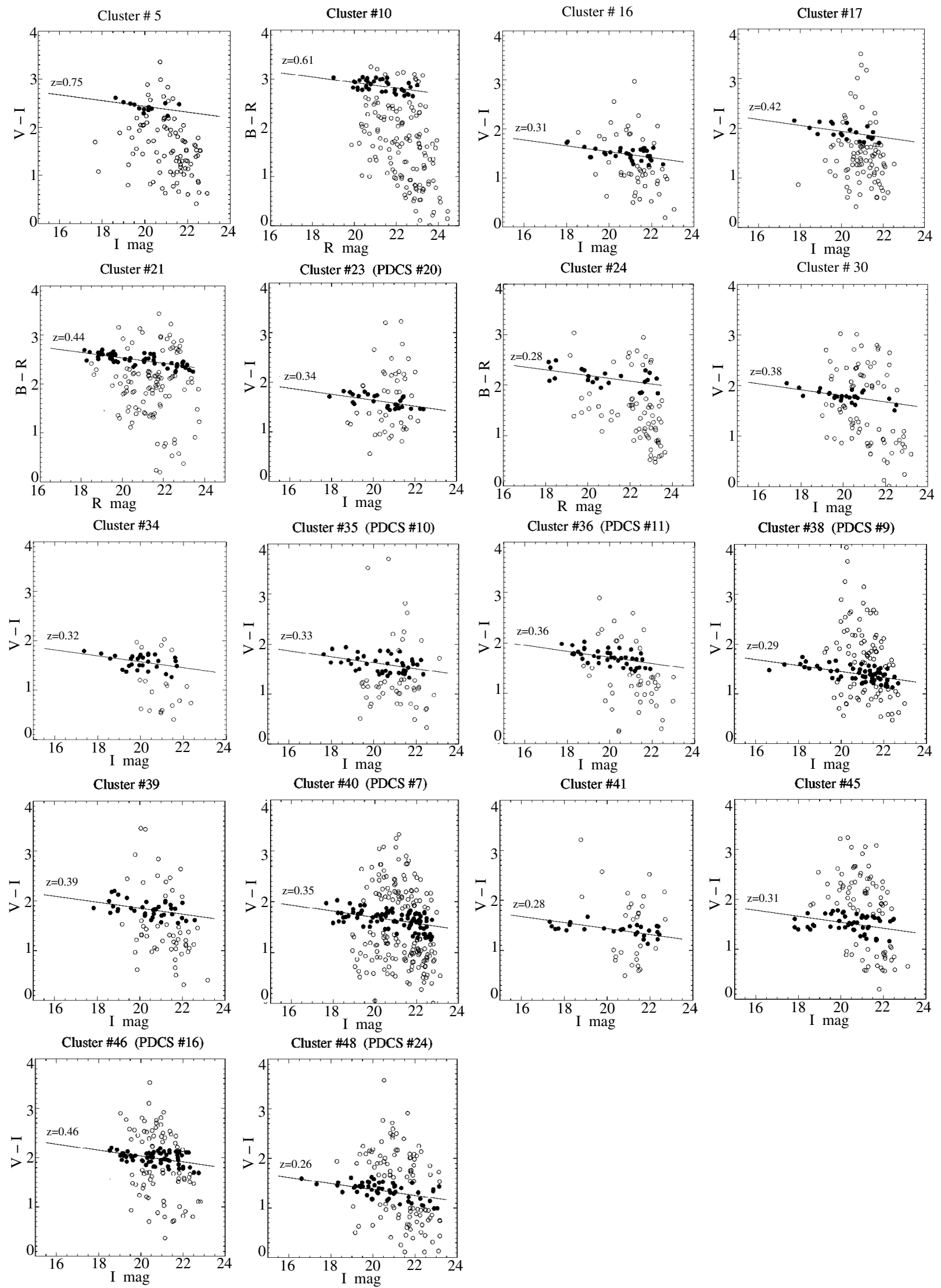

Fig. 3. ETS and redshift estimates of clusters within our survey. This sample includes clusters with ETS in only one of either $(B-R, R)$ or $(V-I, I)$ diagrams. 